\title{
ELECTRICAL INTERFACE FOR A SELF-POWERED MR DAMPER-BASED VIBRATION REDUCTION SYSTEM
}

\author{
Łukasz JASTRZĘBSKI*, Bogdan SAPIŃSKI
}

"Mechanical Engineering and Robotics, Department of Process Control, University of Science and Technology, al. Mickiewicza 30, 30-059 Kraków, Poland

lukasz.jastrzebski83@gmail.com, deep@agh.edu.pl

received 31 March 2016, revised 8 July 2016, accepted 11 July 2016

\begin{abstract}
The study investigates the behaviour of an electrical interface incorporated in a MR damper-based vibration reduction system powered with energy recovered from vibration. The interface, comprising the $R, L$ and $C$ elements, is connected in between the coil in an electromagnetic electric generator and the control coil in the MR damper and its function is to convert the output voltage from the generator. The interface model was formulated and computer simulations were performed to find out how the parameters of the interface should influence the frequency responses of the vibration reduction system.
\end{abstract}

Key words: vibration, electrical interface, MR damper, energy harvesting

\section{INTRODUCTION}

In recent years mechanical systems with energy recovery capability have received a great deal of researchers' attention. Vibration energy is converted into electric energy mostly by the electromagnetic, electrostatic, piezoelectric or magnetostrictive mechanisms. Major developments in the field of energy recovery from mechanical systems to be used for power supply include alternative or conventional low-power energy sources to power the sensors. When the energy receivers are electric-controlled actuators, the generators are needed with significantly larger power outputs.

Typically, power outputs required for supplying (control) of MR dampers in semi-active vibration reduction systems in the automotive industry range from the tenths of watt to several watts. Nearly twenty years before first efforts were made to engineer vibration reduction systems equipped with MR dampers supplied with regenerated energy. Those systems make use of the electromagnetic method of energy conversion based on the Faraday's law. These issues are addressed in more detail in the works of Cho et al. 2005, 2007a, 2007b), Choi and Werely (2009), Wang et al. (2009), Sapiński (2008, 2011), Snamina and Sapiński (2011). Results reported in those papers indicate that energy recovered from vibration is sufficient to power MR dampers and ensure vibration reduction in the neighbourhood of the resonance frequency. This advantage of such systems comes at the cost of increased vibration amplitudes at frequencies above the resonance frequency. To prevent that happening, purpose-built electrical interfaces are incorporated in the system, connected between the generator coil and the control coil in the MR damper (Rosół et al., 2010; Sapiński, 2014; Sapiński et al., 2016).

The model was developed and computer simulations were performed of a semi-active vibration reduction system equipped with a MR damper supplied with energy regenerated from vibration and incorporating an electrical interface made of: $\mathrm{R}$ - resistor, C - capacitor, L - inductor elements, whose operation is based on electric resonance of voltages. The study was undertaken to inves- tigate how the parameters of an electrical circuit formed by the interconnected generator coil, a MR damper coil and a electrical interface should affect the frequency responses of the 1 DOF vibration reduction system, shown schematically in Fig. 1. The source of vibration is a shaker, the vibration reduction system comprises a generator, a spring and a MR damper and the body to be vibro-isolated.

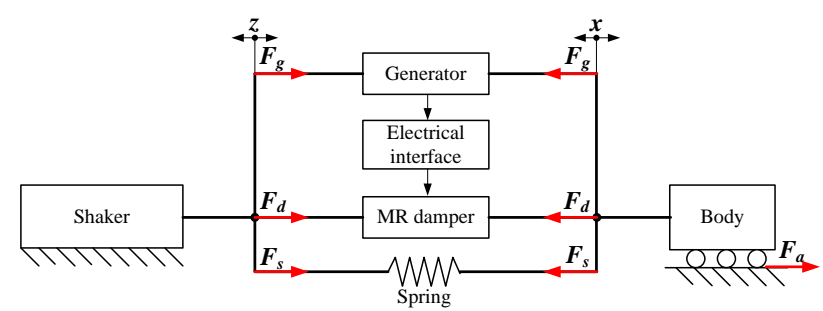

Fig. 1. Semi-active MR damper-based vibration reduction system

\section{MODELLING}

The model of an electrical circuit, comprising a generator coil, an electrical interface and a MR damper control coil, is assumed to be linear. The diagram of an equivalent circuit is shown in Fig. 2.

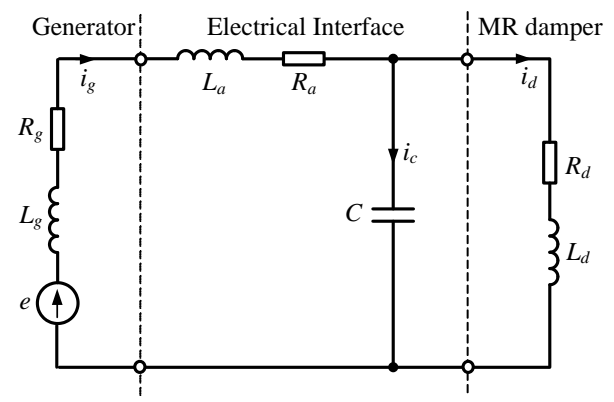

Fig. 2. Equivalent electric circuit 
The input applied is the electromotive force $e$ induced in the generator coil windings with resistance $R_{g}$ and inductance $L_{g}$. The response will be current in the MR damper control coil $i d$. The electrical interface also incorporates an additional coil with resistance $R_{a}$ and inductance $L_{a}$, and a capacitor with capacity $C$. As the generator coil and the interface coil are connected in series, the equivalent resistance of those coils is introduced: $R=R_{g}+R_{a}$ as well as equivalent inductance $L=L_{g}+L_{a}$.

The equation of the circuit shown in Fig. 2 is given as:

$C L_{d} L \frac{d^{3} i_{d}}{d t^{3}}+\left[C\left(L_{d} R+R_{d} L\right)\right] \frac{d^{2} i_{d}}{d t^{2}}+\left(C R_{d} R+L+\right.$ $\left.L_{d}\right) \frac{d i_{d}}{d t}+\left(R+R_{d}\right) i_{d}=e$.

Assuming the relative displacement $y=x-z$, the MR damper force can be expressed by the formula (Guo et al., 2006, Kwok et al., 2006):

$F_{d}=F_{0} \tanh \left[\beta\left(\frac{d y}{d t}+p_{1} y\right)\right]+C_{1}\left(\frac{d y}{d t}+p_{2} y\right)$.

It is assumed that parameters $F_{0}$ and $C_{1}$ are dependent on current $i_{d}$, in accordance with $\mathrm{Eq}(3)$ and (4) whilst the parameters $\beta, \rho_{1}, \rho_{2}$ are not related to current $i_{d .}$.

$F_{0}=b_{1} \cdot i_{d}^{2}+b_{2} \cdot i_{d}+b_{3}$,

$C_{1}=b_{4} \cdot i_{d}^{2}+b_{5} \cdot i_{d}+b_{6}$.

The equation describing the circuit in Fig 1 is written as:

$\frac{d^{2} x}{d t^{2}}=-\frac{1}{m}\left(F_{s}+F_{d}+F_{g}+F_{a}\right)$,

where: $F_{a}=b \cdot d y / d t$ is resistance force in kinematic pairs, $F_{s}=k \cdot y$ is elasticity force, $F_{g}=K \cdot \dot{l}_{g}$ is magnetic force in the generator. It is assumed that electromotive force $e$ and current $i_{d}$ in the generator coil are expressed by respective formulas:

$e=\kappa \frac{d y}{d t}$,

$i_{g}=i_{d}+C R_{d} \frac{d i_{d}}{d t}+C L_{d} \frac{d^{2} i_{d}}{d t^{2}}$,

\section{SIMULATION}

Simulations were performed in the MATLAB/Simulink environment. The values of the MR damper model parameters were quoted from the work by (Sapiński et al., 2011): $\beta=50 \mathrm{~N} \cdot \mathrm{s} / \mathrm{m}, \rho_{1}=41 / \mathrm{s}$, $\rho_{2}=0.2 \quad 1 / \mathrm{s}, \quad b_{1}=3415.7 \quad \mathrm{~N} / \mathrm{A}^{2}, \quad b_{2}=93.32 \quad \mathrm{~N} / \mathrm{A}, \quad b_{3}=74.487 \quad \mathrm{~N}$, $b_{4}=2534.1 \mathrm{~N} \cdot \mathrm{s} / \mathrm{A}^{2} \mathrm{~m}, b_{5}=19.55 \mathrm{~N} \cdot \mathrm{s} / \mathrm{A} \cdot \mathrm{m}, b_{6}=643.1 \mathrm{~N} \cdot \mathrm{s} / \mathrm{m}$. The remaining parameters were: body mass $m=103 \mathrm{~kg}$, stiffness coefficient $k=90000 \mathrm{~N} / \mathrm{m}$, damping ratio $b_{1}=50 \mathrm{~N} \cdot \mathrm{s} / \mathrm{m}$, electric constant of the generator $k=18.6 \mathrm{~V} \cdot \mathrm{s} / \mathrm{m}$, inductance of a MR damper control coil $L_{d}=100 \mathrm{mH}$, MR damper coil resistance $R_{d}=5 \Omega$, equivalent inductance $L=30 \mathrm{mH}$, equivalent resistance $R=0.4 \Omega$, capacitor capacity $\mathrm{C}=47 \mathrm{mF}$. The simulation procedure involved three stages, the frequency characteristics were obtained for the equivalent system shown in Fig. 2 and for the vibration reduction system shown in Fig. 1, with and without the electrical interface.

\subsection{Electrical interface}

Fig. 3 shows how the variations of resistance $R$, capacitance $C$, inductance $L$ and resonance frequency $f_{r}$ of the electric circuit affect the frequency characteristics of the magnitude frequency response $\left|G_{i i}(22 \pi f)\right|=|I /(2 \pi f) / E(j 2 \pi f)|$.

a)

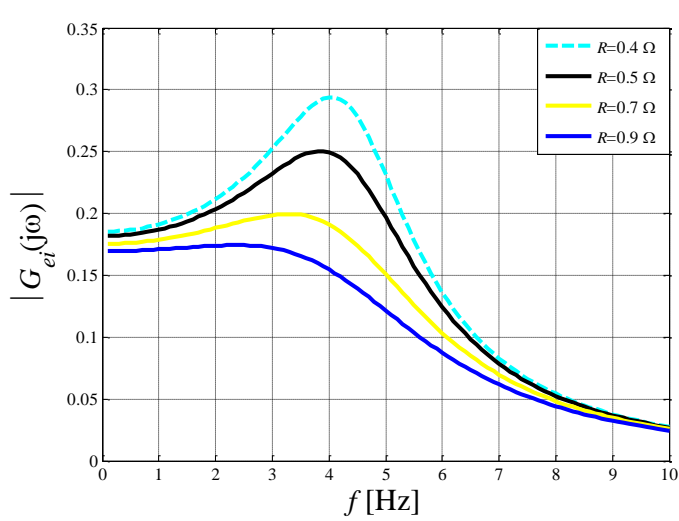

b)

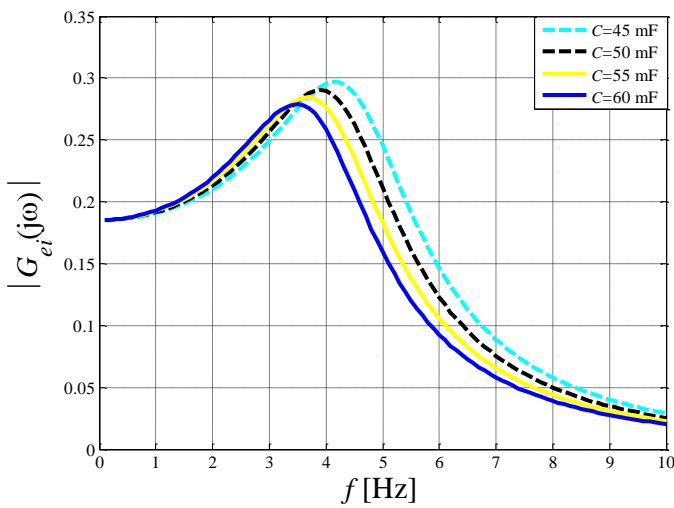

c)

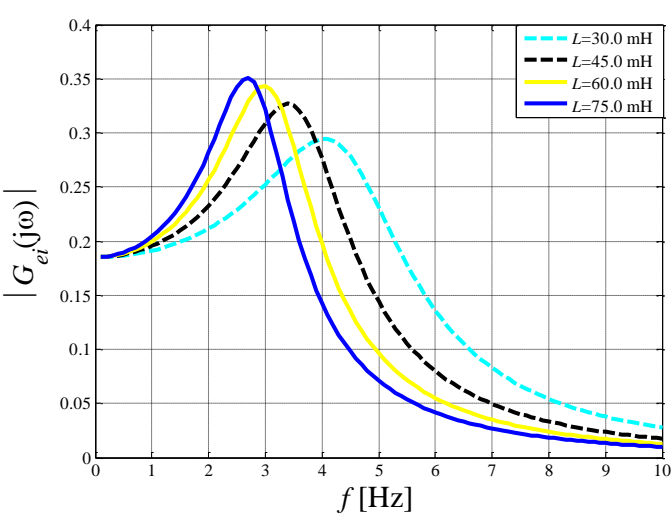

d)

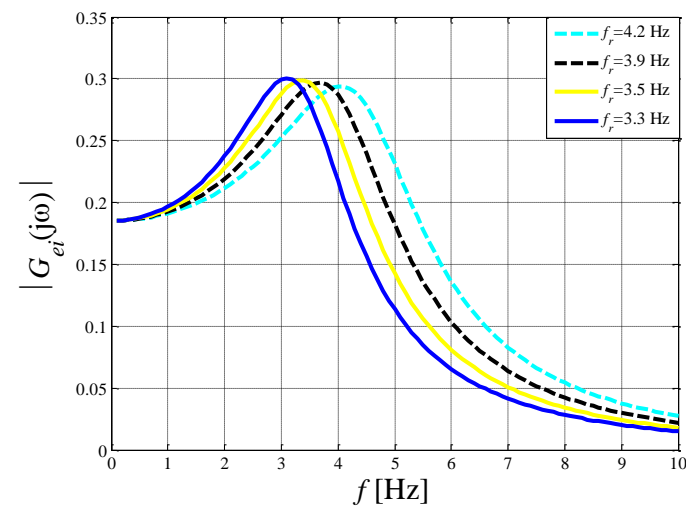

Fig. 3. Frequency response $\left|G_{e}(22 \pi f)\right|:$ a) $R_{g}=v a r$, b) $C=v a r$, c) $L=$ var, d) $f_{l}=v a r$ 
It is apparent that:

- increase of resistance $R$ and capacitor capacity $C$ causes the resonance frequency $f_{r}$ to be reduced and the maximum value of $\left|G_{e i}(2 \pi f)\right|$ to be lowered (Fig. 3a, b);

- for resistance $R>0.9 \Omega$, the plot $\left|G_{e}(j 2 \pi f)\right|$ does not have a maximum for the frequency $f_{r}$ (Fig. 3a);

- increasing the inductance $L$ results in reduction of resonance frequency $f_{r}$, at the same time the maximum value of $\left|G_{e i}(j 2 \pi f)\right|$ will increase (Fig. 3c);

- simultaneous increase of the values of $C$ and $L$ leads to the reduction of frequency $f_{r}$ whilst the maximum value of $\left|G_{e i}(j 2 \pi f)\right|$ remains unchanged (Fig. 3d);

- simultaneous increase of inductance $L$ and reduction of capacity $C$ should result in an increase of the maximum value $\left|G_{e i}(2 \pi f)\right|$ whilst the frequency $f_{r}$ remains constant.

The change of frequency $f_{r}$ whilst the maximal value $\left|G_{e i}(j 2 \pi f)\right|$ remain constant allows this frequency to be tuned to the resonance frequency $f_{r m}$ of the system shown in Fig. 1. The value of $\left|G_{e i}(2 \pi f)\right|$ for the frequency $f_{r}$ is associated with the quality factor of the circuit $Q=\sigma / R$, which is proportional to wave impedance $\sigma=\sqrt{ } L / C$. With decreasing $\sigma$, the characteristics tend to change, like with increasing resistance $R$ (Fig. 3a).

\subsection{Vibration reduction system without an electrical interface}

Computer simulations were performed to investigate the behaviour of the system shown in Fig. 1 configured such that the MR damper control coil was directly supplied from the generator. The kinematic input excitation $z$ was applied, with the amplitude $A=3.5 \mathrm{~mm}$ and frequency from the range $(0.1,10) \mathrm{Hz}$. The purpose of the simulation procedure was to find the displacement transmissibility $T_{x z}$ given by the formula (8), rms values of electromotive force $E_{r m s}$ and current level in the control coil $I_{r m s}$, as well as the rms value of the damper force $F_{d}$.

$T_{x Z}=\frac{X_{r m s}}{Z_{r m s}}=\frac{\frac{1}{t} \sqrt{\int_{0}^{t} x(t)^{2} d t}}{\frac{1}{t} \sqrt{\int_{0}^{t} z(t)^{2} d t}}$

Simulation data are compiled in Figs 4-7, plotting the frequency characteristics. Comparison was made between two cases: when the control coil was not supplied (P-passive system) and when the control coil was supplied directly from the generator (SP-self powered system).

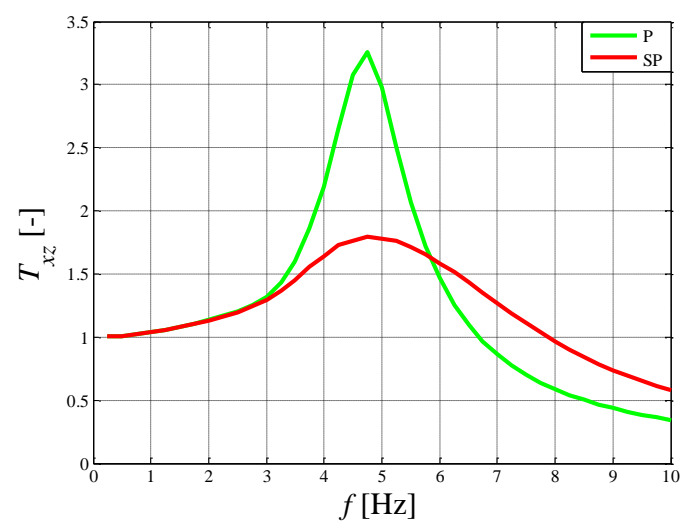

Fig. 4. Transmissibility vs frequency
Coefficients $T_{x z}$ (see Fig. 4) for the systems $P$ and SP reach the maximal value at the resonance frequency $f_{r m}=4.75 \mathrm{~Hz}$ and are equal to $T_{x z}=3.26$ and $T_{x z}=1.8$, respectively. In the system SP the value of $T_{x z}$ decreases in the range $(3,5.75)$ in relation to the system $P$ and increases when $>5.75 \mathrm{~Hz}$. $E_{r m s}$ in the system SP increases with frequency, whilst in the system $P$ it reaches its maximum value for $f=5 \mathrm{~Hz}$ (Fig. 5). Decreased value of $I_{\text {ms }}$ for frequency $f>8 \mathrm{~Hz}$ is attributed to the increase of the control coil's impedance (Fig. 6). The force $F_{d}$ reaches its maximum value at frequency $f=5 \mathrm{~Hz}$ in the system $P$ and at $f=9 \mathrm{~Hz}$ in the system SP (Fig. 7).

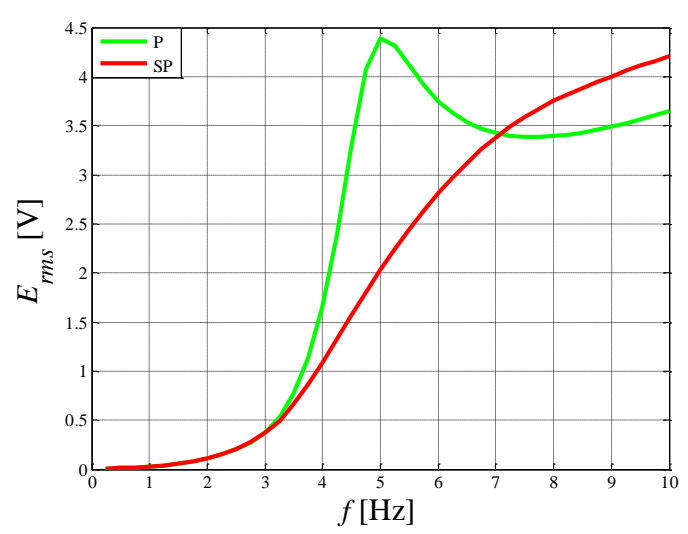

Fig. 5. Electromotive force vs frequency

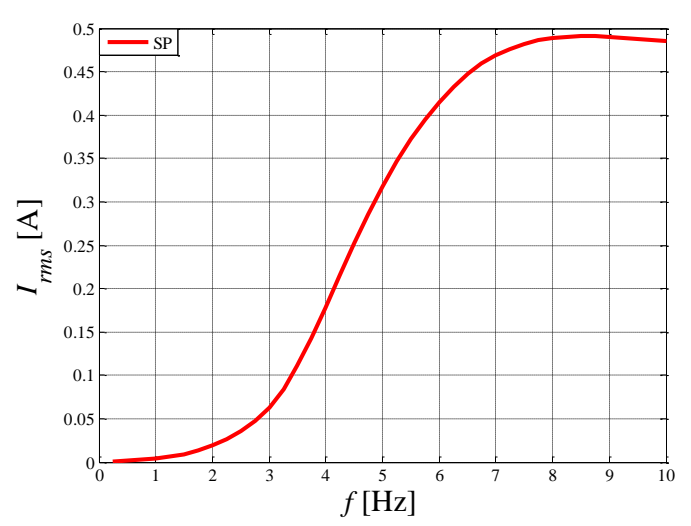

Fig. 6. Current vs frequency

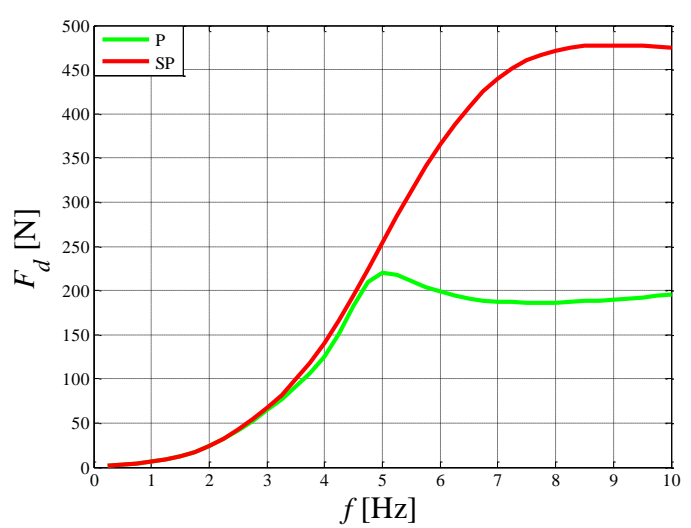

Fig. 7. Force vs frequency 


\subsection{Vibration reduction system incorporating an electrical interface}

Simulations were performed to investigate the behaviour of the system shown in Fig. 1, configured such that an electrical interface is connected between the generator coil and the MR damper control coil (system SPI). The receiver's parameters considered in the simulation procedure were constant $\left(R_{d}, L_{d}\right)$, and the parameters of the applied input were those given in section 3.2. The main objective was to establish how the parameters $R, L, C$, the generator's electric constant $k$ and resonance frequency $f_{r}$ should influence the frequency characteristics of the system. Simulation data are summarised in Figs 8-27.

The analysis of plots shown in Figs 8-11 reveals that increasing the generator's electric constant $k$ leads to:

- decrease of $T_{x z}$ in the neighbourhood of resonance frequency (Fig. 8);

- increase of electromotive force value $E_{r m s}$ and current $I_{r m s}$ (Figs 9 , 10);

- increase of the force value $F_{d}$ in the interval $(4,10) \mathrm{Hz}$ (Fig. 11).

For the maximum value of the electric constant considered in the simulation procedure $k=20 \mathrm{~V} \cdot \mathrm{s} / \mathrm{m}$, we observe:

- the anti-resonance effect at the same frequency $\left(f_{a}=4.75 \mathrm{~Hz}\right)$ at which the mechanical resonance $f_{r m}$ is registered in the systems $P$ and SP;

- nearly 3-fold reduction of the value of $T_{x z}$ at frequency $f_{a}$ in relation to that registered for system $\mathrm{P}$ and 1.5-fold reduction of $T_{x z}$ in relation to SP;

- increased the $T_{x z}$ value in the frequency range $(6,8) \mathrm{Hz}$, in relation to that registered in the system $P$.

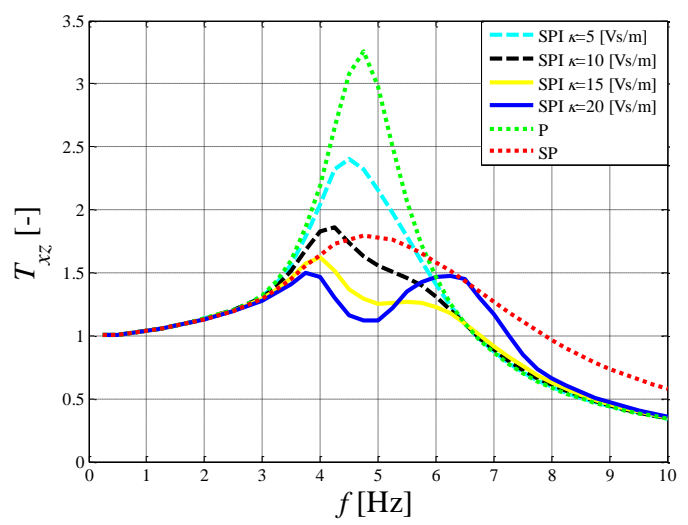

Fig. 8. Transmissibility vs frequency

The lowest value of $T_{x z}$ in the investigated frequency range is registered for the system SPI, its electric constant being $k=15$ $\mathrm{V} \cdot \mathrm{s} / \mathrm{m}$. At the same time the value of $T_{x z}$ tends to decrease in the neighbourhood of resonance frequency and remains on the level similar to that registered for the system $\mathrm{P}$ at $>-6.25 \mathrm{~Hz}$. A capacitor with capacity $C$ incorporated in the electrical interface lowers its impedance with increasing input frequency $f$, causing the current level $I_{m s}$ to decrease in a control coil connected in parallel to the condenser at $f>f_{r m}$ (Fig. 10). In the system SPI the value of $T_{x z}$ is reduced at frequencies in excess of $f_{m}$, when compared to SP.

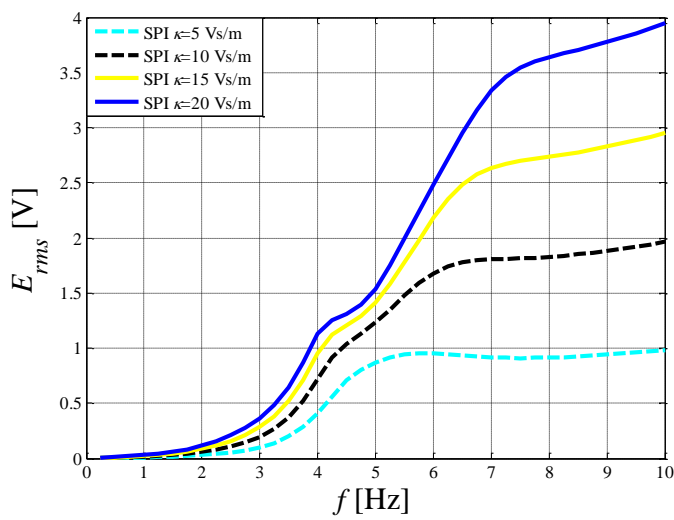

Fig. 9. Electromotive force vs frequency

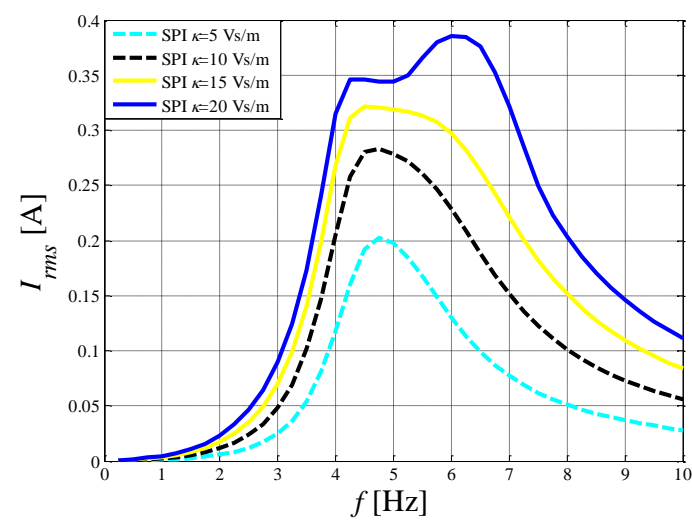

Fig. 10. Current vs frequency

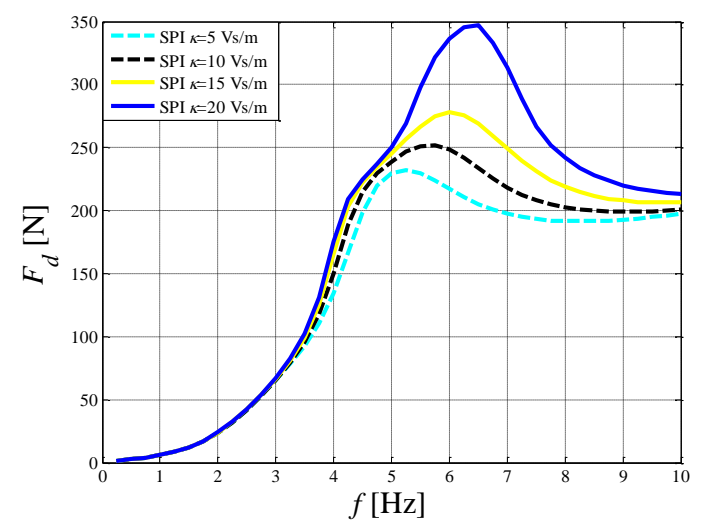

Fig. 11. Force vs frequency

The analysis of plots shown in Fig 12-15 reveals that decreasing the resistance $R$ will lead to:

- lowering the value of $T_{x z}$ in the neighbourhood of resonance frequency (Fig. 12);

- lowering the value of $E_{m s}$ in the frequency range $(4,7) \mathrm{Hz}$ (Fig. 13);

- increasing the value of $I_{m s}$ and force $F_{d}$ at $\ngtr 4 \mathrm{~Hz}$ (Figs 14, 15); 


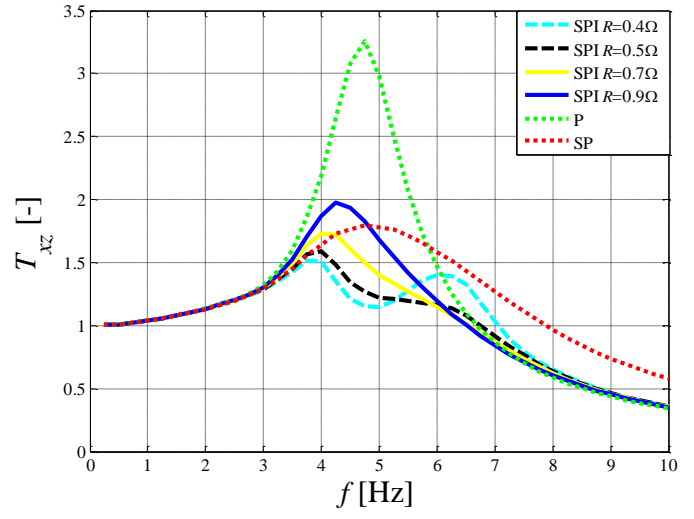

Fig. 12. Transmissibility vs frequency

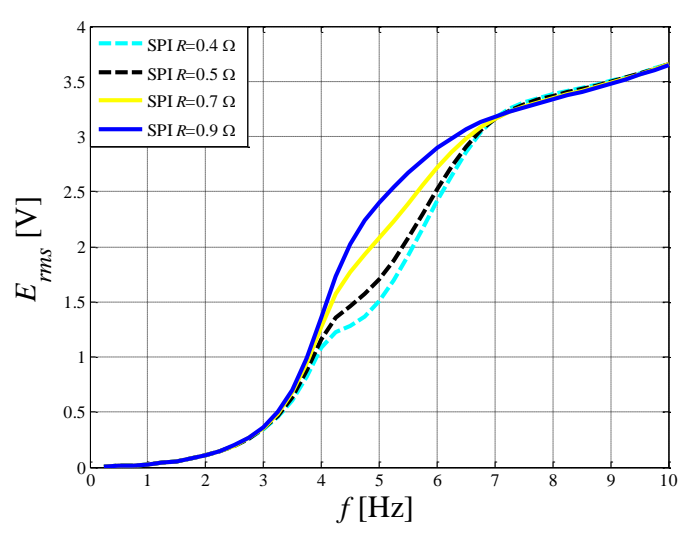

Fig. 13. Electromotive force vs frequency

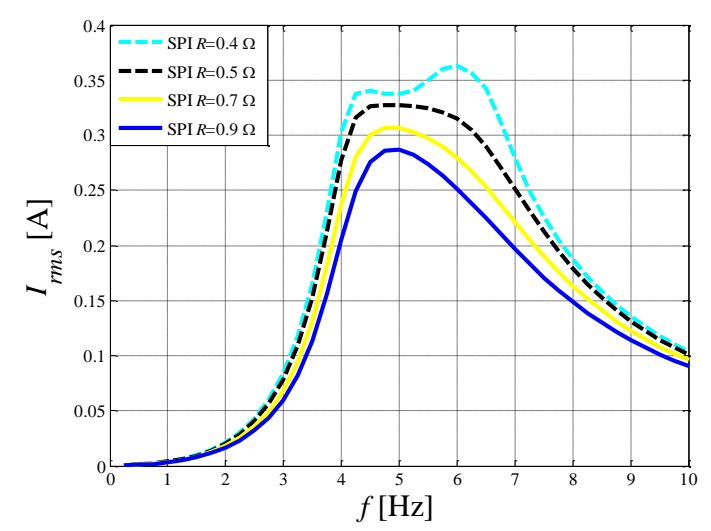

Fig. 14. Current vs frequency

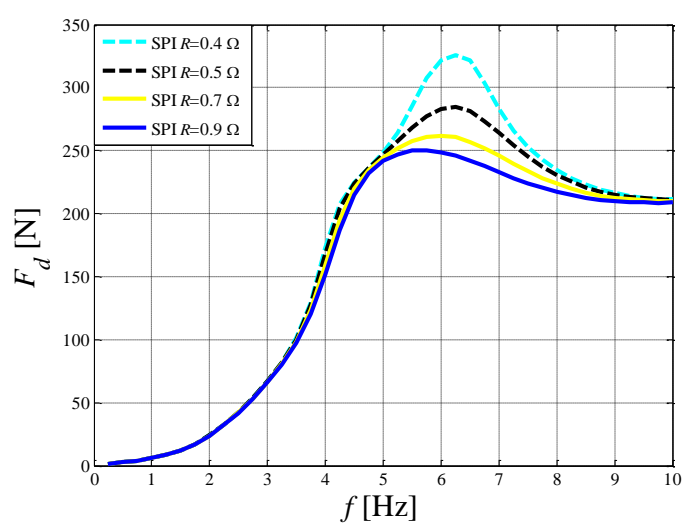

Fig. 15. Force vs frequency
When resistance $R=0.4 \Omega$, the anti-resonance effect will arise $\left(f_{a}=4.75 \mathrm{~Hz}\right.$ ), at the same time the value of $T_{x z}$ registered in the frequency range $(6,8) \mathrm{Hz}$ is higher than in the circuit $P$. In the investigated frequency range and at resistance $R=0.5 \Omega$ the value of $T_{x z}$ in the circuit SPI is lower in relation to the values registered for $\mathrm{P}$ and SP.

The analysis of plots in Figs 16-19 reveals that reducing the condenser capacity $C$ results in:

- decrease of the value of $T_{x z}$ (Fig 16) at the anti-resonance frequency $f_{a}$, at the same time this frequency will increase;

- decrease of the $E_{m s}$ value (Fig 17) in the frequency range $(4,8)$ $\mathrm{Hz}$;

- increase of the $I_{r m s}$ value (Fig. 18) and of force $F_{d}$ (Fig. 19) in the frequency range $(4,10) \mathrm{Hz}$.

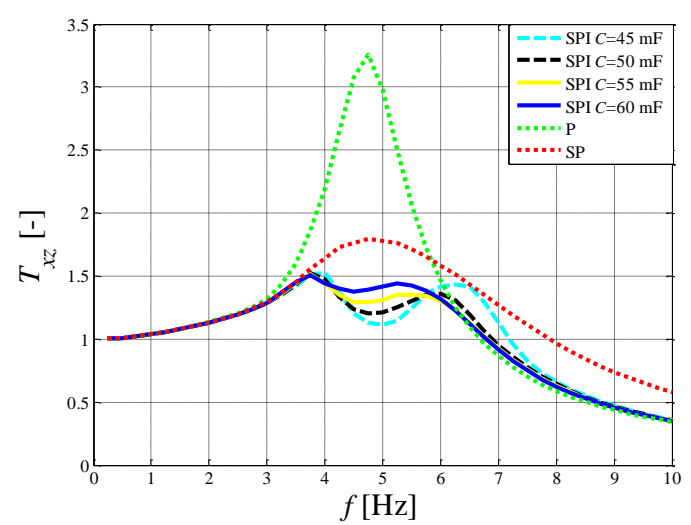

Fig. 16. Transmissibility vs frequency

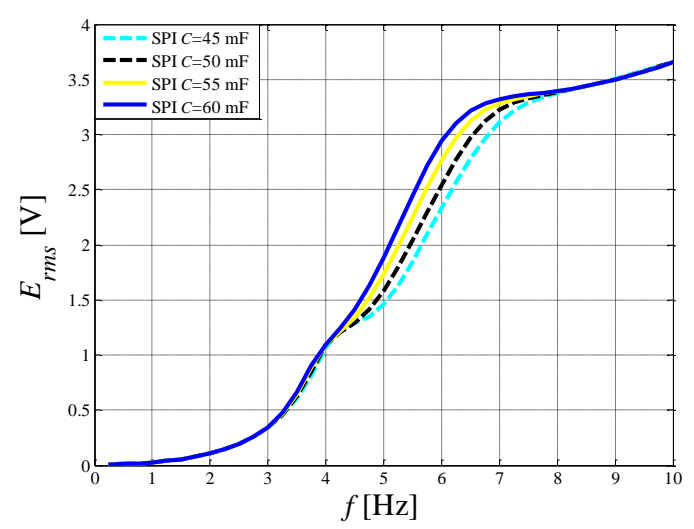

Fig. 17. Electromotive force vs frequency

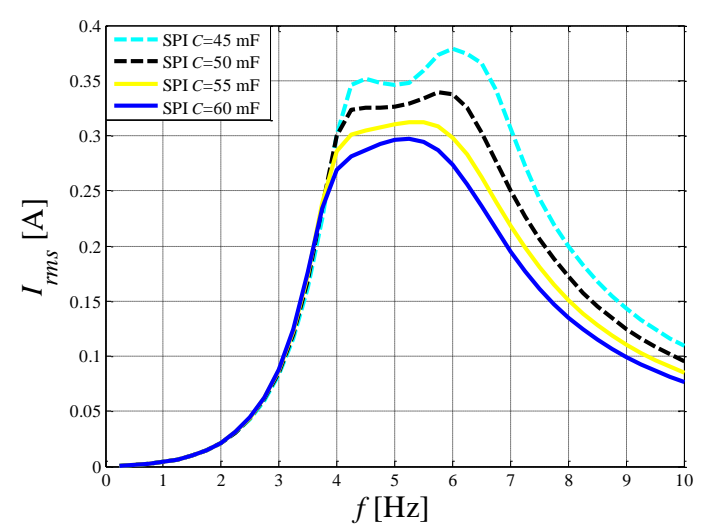

Fig. 18. Current vs frequency 


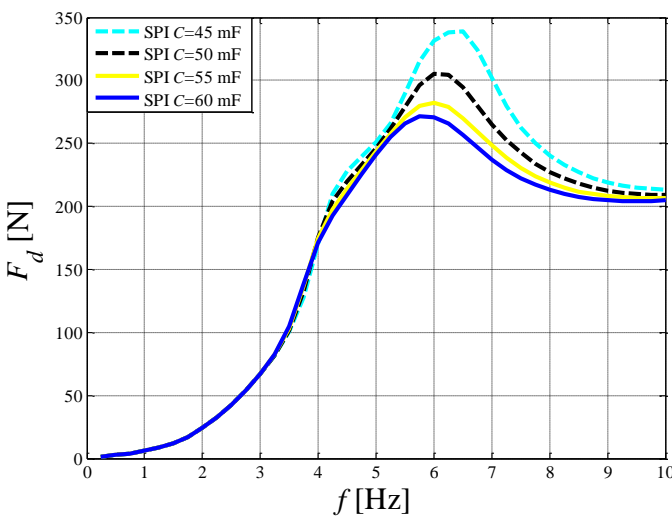

Fig. 19. Force vs frequency

For capacitor capacity $\mathrm{C}=45 \mathrm{mF}$, the value of $T_{x z}$ registered in the frequency range $(6,8) \mathrm{Hz}$ is higher than in the circuit $\mathrm{P}$. The lowest value of $T_{x z}$ in the investigated frequency range is registered in the circuit SPI for $C=50 \mathrm{mF}$ (Fig. 16).

The analysis of plots in Figs 20-23 reveals that reducing the inductance $L$ will lead to:

- decrease of $T_{x z}$ value in the frequency range $(3,6) \mathrm{Hz}$ (Fig. 20);

- increase of resonance frequency $f_{r m}$ when $L>45 \mathrm{mH}$ and increase of anti-resonance frequency $f_{a}$ when $L \leq 45 \mathrm{mH}$;

- lowering the value of $E_{r m s}$ in the frequency range $(4,8) \mathrm{Hz}$ (Fig. 21);

- increase of $I_{r m s}$ and force $F_{d}$ in the frequency range $(3,10) \mathrm{Hz}$ (Figs 22, 23);

- increase of the frequency $f$ at which the force $F_{d}$ reaches its maximum value.

When inductance $L=30 \mathrm{mH}$, the value of $T_{x z}$ in the frequency range $(6,8) \mathrm{Hz}$ is increased. The lowest values of $T_{x z}$ in the investigated frequency range, compared to the circuits $P$ and $S P$, were registered for $L=45 \mathrm{mH}$. Plots in Figs $24-27$ illustrate the effects of varying the resonance frequency of the electrical circuit $f_{r}$, while the quality factor $Q$ of the circuit remains unchanged. The anti-resonance effect (Fig 24) occurs for all investigated frequencies $f_{r}$.

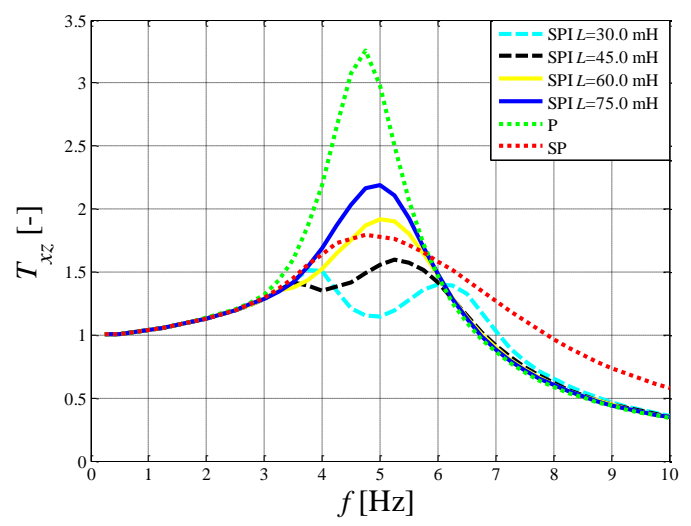

Fig. 20. Transmissibility vs frequency

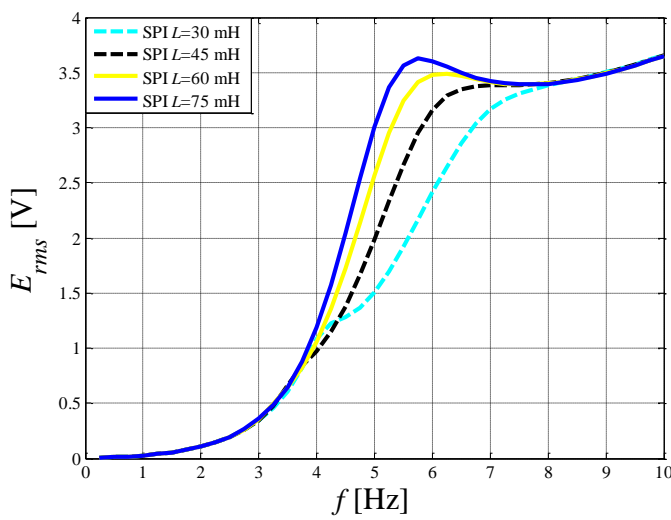

Fig. 21. Electromotive force vs frequency

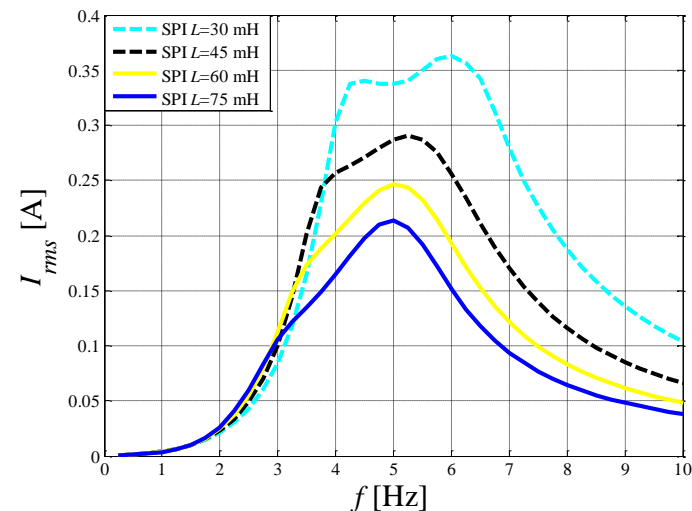

Fig. 22. Current vs frequency

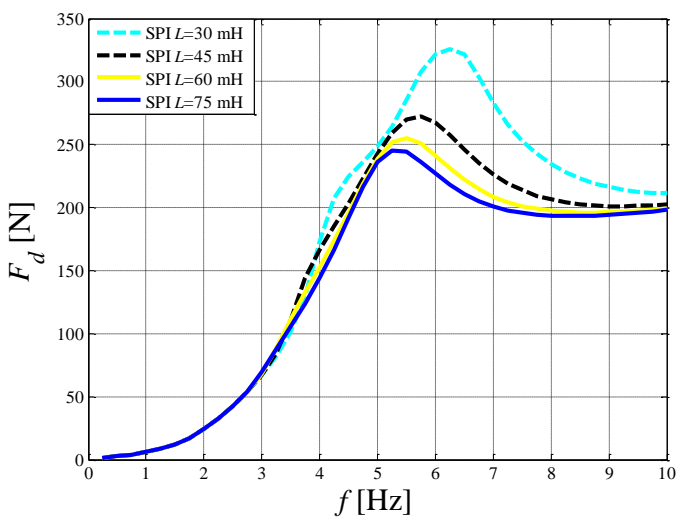

Fig. 23. Force vs frequency

The analysis of plots in Figs 24-27 reveals that increase of frequency $f_{r}$ will lead to:

- increase of anti-resonance frequency $f_{a}$ of the mechanical system and reduction of $T_{x z}$ value at $f_{a}$ (Fig. 24);

- reduction of the $E_{\text {rms }}$ value in the frequency range $(4,8) \mathrm{Hz}$ (Fig. 25);

- increasing the value of $I_{m s}$ and force $F_{d}$ in the frequency range $(4,10) \mathrm{Hz}$ (Figs 26, 27). 


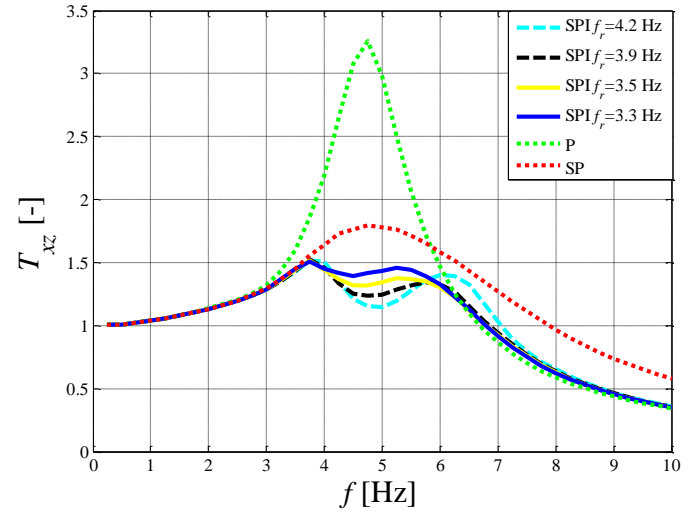

Fig. 24. Transmissibility vs frequency

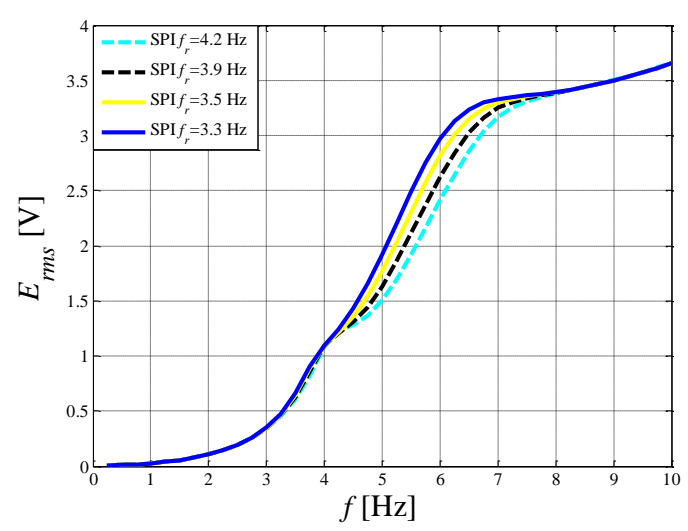

Fig. 25. Electromotive force vs frequency

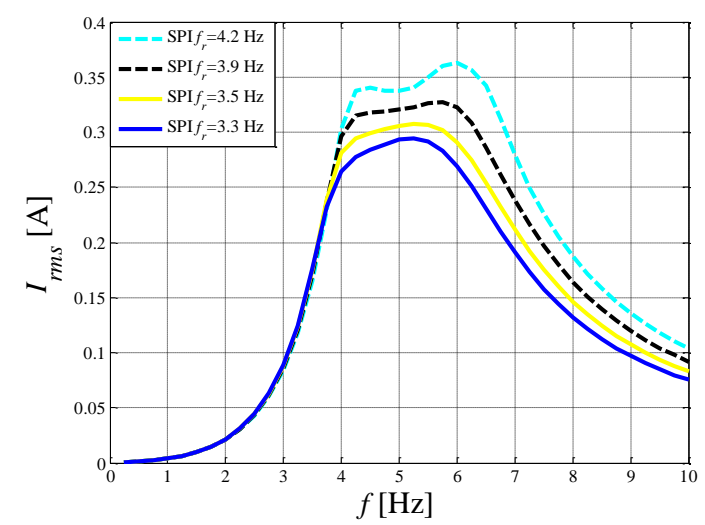

Fig. 26. Current vs frequency

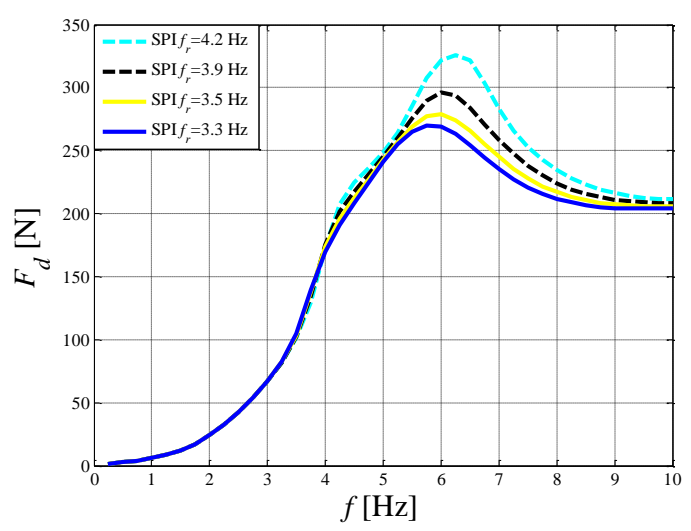

Fig. 27. Force vs frequency
It appears that the value of $T_{x z}$ registered in the frequency range $(6,7.5) \mathrm{Hz}$ is higher than in the system $P$ when $f_{r}=4.2 \mathrm{~Hz}$. Compared to $\mathrm{P}$ and $\mathrm{SP}$, the lowest values of $T_{x z}$ in the investigated frequency range are registered in the system SPI, where the resonance frequency of the electric circuit is $f_{r}=3.9 \mathrm{~Hz}$.

\section{SUMMARY}

The study summarises the simulations of a model of a semiactive vibration reduction system comprising an MR damper, incorporating an electrical interface whose operating principle is based on voltage resonance. The interface, comprising the $R, L$, $C$ elements, is connected between the generator coil and the MR damper control coil. The simulation procedure was performed to investigate the effect of the interface parameters on frequency characteristics of the vibration reduction system. The analysis of thus obtained frequency characteristics leads us to the following conclusions:

- the magnitude frequency response $\left|G_{e i}(j 2 \pi f)\right|$ reaches its maximal value at the resonance frequency of the equivalent circuit when the equivalent resistance becomes $R<0.9 \Omega$;

- simultaneous change of the parameters $L$ and $C$ allows the resonance frequency of the equivalent circuit to be tuned to resonance frequency of the vibration reduction system;

- the maximal value of the magnitude frequency response $\left|G_{e}(j 2 \pi f)\right|$ at the resistance frequency is associated with the quality factor of the equivalent circuit;

- transmissibility in the system SP has lower values than in system $P$ in the neighbourhood of resonance frequency whilst at frequencies in excess of resonance frequency its values are higher;

- in the system SPI the transmissibility values are lower when compared to $\mathrm{P}$ and $\mathrm{SP}$ in the entire frequency range;

- voltage resonance in the equivalent circuit gives rise to the anti-resonance effects in the vibration reduction system, revealed by the presence of minimum in the transmissibility vs frequency plot;

- the anti-resonance effect may occur at the same frequency at which the mechanical resonance will occur in the system $P$ and SP.

The model of the electrical interface considered in the paper assumes linearity of $R, L, C$ elements and does not take into account the influence of eddy currents. The problem of eddy currents which occur both in the magnetic circuit of the MR damper and the generator will be the subject of further authors' research.

\section{REFERENCES}

1. Cho S. W., Jung H. J., Lee I. W. (2005), Smart passive system based on a magnetorheological damper, Smart Materials and Structures, 14, 707-714.

2. Cho S. W., Jung H. J., Lee I. W. (2007a), Feasibility study of smart passive control system equipped with electromagnetic induction device, Smart Materials and Structures, 16, 2323-2329.

3. Choi K. M., Jung H. J., Lee I. W., Cho S. W. (2007b), Feasibility study of an MR damper-based smart passive control system employing an electromagnetic induction device, Smart Materials and Structures, 16, 2323า-9.

4. Choi Y. T., Werely N. M. (2009), Self-powered magnetorheological dampers, Journal of Vibration Acoustics, 131, 44-50. 
5. Guo S., Yang S., Pan C. (2006), Dynamic modeling of magnetorheological damper behaviors, Journal of Intelligent Material Systems and Structures, 17, 1, 3-14.

6. Kwok N.M, Ha Q.P., Nguyen T.H., Li J., Samali B. (2006), A novel hysteretic model for magnetorheological fluid dampers and parameter identification using particle swarm, Sensors and Actuators $A, 132,441-451$.

7. Rosół M., Sapiński B., Jastrzębski $Ł$. (2010), Laboratory testing of signal conditioning systems in an electromagnetic generator to power-supply an MR damper, Measurement, Automation, Monitoring, 56(10), 1228-1233 (in Polish).

8. Sapinski B. (2008), An experimental electromagnetic induction device for a magnetorheological damper, Journal of Theoretical and Applied Mechanics, 46(4), 933-947.

9. Sapiński B. (2011), Experimental study of a self-powered and sensing MR-damper-based vibration control system, Smart Materials and Structures, 20, 105007.
10. Sapiński B. (2014), Energy-harvesting linear MR damper: prototyping and testing, Smart material and Structures, 23, 035021.

11. Sapiński B., Jastrzębski Ł., Węgrzynowski M. (2011), Modelling of a self-powered vibration reduction system, Modelling in Engineering, 10(41), 353-362 (in Polish).

12. Sapiński B., Rosół M., Węgrzynowski M. (2016), Evaluation of an energy harvesting $\mathrm{mr}$ damper-based vibration reduction systemstem, Journal of Theoretical and Applied Mechanics, 54(2), 333-344.

13. Snamina J., Sapinski B. (2011), Energy balance in self-powered MR damper-based vibration reduction system, Bulletin of the Polish Academy of Sciences Technical Sciences, 59(1), 75-80.

14. Wang D. H., Bai X. X., Liao W H. (2009), Principle, design and modeling of an integrated relative displacement magnetorheological damper based on electromagnetic induction, Smart Materials and Structures, 18, 095025.

This work is supported by AGH University of Science and Technology under research program No. 11.11.130.958. 